\title{
Superspace Formulation of the Chern Character of a Theta-Summable Fredholm Module
}

\author{
Andrzej Lesniewski ${ }^{1 \star}$, Konrad Osterwalder ${ }^{2}$ \\ 1 Lyman Laboratory of Physics, Harvard University, Cambridge, MA 02138, USA \\ 2 Department of Mathematics, ETH, 8092 Zürich, Switzerland
}

Received: 10 February 1994

\begin{abstract}
We apply the concepts of superanalysis to present an intrinsically supersymmetric formulation of the Chern character in entire cyclic cohomology. We show that the cocycle condition is closely related to the invariance under supertranslations. Using the formalism of superfields, we find a path integral representation of the index of the generalized Dirac operator.
\end{abstract}

\section{Introduction}

I. A. The purpose of this note is to present an intrinsically supergeometric formulation of the Chern character of [7] in entire cyclic cohomology [3]. The concept of a Fredholm module is closely related to the structure of supersymmetric quantum theory. The construction of the Chern character associated with a $\Theta$-summable Fredholm module presented in [7] uses in an essential way ideas adopted from supersymmetric quantum field theory. The $n^{\text {th }}$ component of the Chern character is written as a certain finite temperature $(n+1)$-point Schwinger function integrated over an $n$-simplex. The physical interpretation of the closedness of the Chern character under Connes' coboundary operator $\partial$ remained, however, unclear.

The construction of this paper is based on the simple observation that a more natural form of the Chern character arises if the integrals over simplexes are replaced by Berezin integrals over supersimplexes. (A supersimplex is a superdomain whose base is an ordinary simplex.) This makes the supersymmetric nature of the Chern character transparent. We find that the Chern character is invariant under the (1|1)-dimensional supergroup of translations in the time direction and in one extra fermionic direction. It is the invariance under supertranslations in the fermionic direction which is equivalent to the closedness of the Chern character under $\partial$. Furthermore, this form of the Chern character lends itself well to a path integral representation. This should be a useful technical tool in studying the topological properties of Fredholm modules arising in quantum field theory. Our discussion

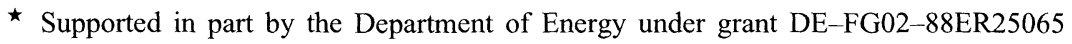


of this idea has a preliminary character and is based on a number of technical assumptions whose validity needs to be established in each situation separately.

I.B. The paper is organized as follows. Section II contains the superspace formulation of the Chern character. In Sect. III, we discuss the relation between the cocycle condition satisfied by the Chern character and its supertranslation invariance. Finally, in Sect. IV we discuss the conditions under which the Chern character and index of the generalized Dirac operator have natural superpath integral representations.

I.C. There are several different definitions of a supermanifold available. For the purpose of this paper, it would not matter which one is used. For convenience, we adopt the Berezin-Leites-Kostant definition, see e.g. [1]. A supermanifold $\mathscr{X}$ is thus a locally trivial ringed space whose structure sheaf is isomorphic to the tensor product of the sheaf of smooth functions on a manifold $X$ (called the base of $\mathscr{X}$ ), and a Grassmann algebra $\bigwedge(E)$ over a vector space $E$. We denote the superalgebra of global sections of this sheaf by $C^{\infty}(\mathscr{X})$, and refer to a set of (even and odd) generators of this superalgebra as the coordinates of a "point" of $\mathscr{X}$. By $\widehat{\otimes}_{\pi}$ we denote the $\mathbb{Z}_{2}$-graded, completed projective tensor product of sheaves. Structure sheaves of supermanifolds are sheaves of nuclear vector spaces, and so using $\widehat{\otimes}_{\pi}$ is convenient as it leads to natural functorial properties of cartesian products of supermanifolds.

\section{The Chern Character}

II. $A$. Let $\mathscr{A}$ be a unital $\mathrm{C}^{*}$-algebra. Recall [2] that a $\Theta$-summable Fredholm module over $\mathscr{A}$ is a triple $(\mathscr{H}, Q, \rho)$, consisting of a $\mathbb{Z}_{2}$-graded Hilbert space $\mathscr{H}$, a selfadjoint operator $Q$ on $\mathscr{H}$ which is odd with respect to the $\mathbb{Z}_{2}$-grading of $\mathscr{H}$, and a grading preserving *-homomorphism $\rho$ of $\mathscr{A}$ to the $\mathrm{C}^{*}$-algebra $\mathscr{L}(\mathscr{H})$ of bounded linear operators on $\mathscr{H}$. For convenience of notation, we suppress $\rho$ in all formulas below. We require that

- the subalgebra $\mathscr{A}_{1}:=\{a \in \mathscr{A}:\|[Q, a]\|<\infty\}$ is dense in $\mathscr{A}$;

- for all $\beta>0$,

$$
\operatorname{Tr}\left(\exp \left(-\beta Q^{2}\right)\right)<\infty
$$

Throughout this paper we will make the simplifying assumption that $\mathscr{A}$ is trivially graded meaning that all elements of $\mathscr{A}$ are even. By $\Gamma$ we denote the grading operator on $\mathscr{H}$. It satisfies the properties $\Gamma^{2}=I$ and $\Gamma^{*}=\Gamma$. By $Q_{+}:=P_{-} Q P_{+}$, where $P_{ \pm}:=\frac{1}{2}(I \pm \Gamma)$, we denote the restriction of $Q$ to the even subspace of $\mathscr{H}$. Finally, by $\operatorname{Tr}(A)$ we denote the trace of $A \in \mathscr{L}(\mathscr{H})$.

A $\Theta$-summable Fredholm module defines a fundamental cohomology class in the entire cyclic cohomology of $\mathscr{A}$, the Chern character [3]. In the representation of [7] and [5], the Chern charater is a sequence of $(n+1)$-linear functionals on $\mathscr{A}, \tau^{\beta}=\left\{\tau_{n}^{\beta}\right\}_{n=0}^{\infty}$ constructed as follows. For $A_{0}, \ldots, A_{n} \in \mathscr{L}(\mathscr{H})$ we define

$$
F_{n}^{\beta}\left(A_{0}, A_{1}, \ldots, A_{n}\right):=\beta^{-n / 2} \int_{\sigma_{n}^{\beta}} \operatorname{Tr}\left(A_{0} A_{1}\left(t_{1}\right) \ldots A_{n}\left(t_{n}\right) \exp \left(-\beta Q^{2}\right)\right) d t,
$$

where $A(t):=\exp \left(-t Q^{2}\right) A \exp t Q^{2}$ is the operator $A$ "at the Euclidean time $t$ " [8], and where $\sigma_{n}^{\beta}:=\left\{\left(t_{1}, \ldots, t_{n}\right) \in \mathbb{R}^{n}: 0<t_{1}<\ldots<t_{n}<\beta\right\}$ is the $n$-simplex of 
length $\beta$. We then set for $a_{0}, \ldots, a_{n} \in \mathscr{A}$,

$$
\tau_{n}^{\beta}\left(a_{0}, a_{1}, \ldots, a_{n}\right):=F_{n}^{\beta}\left(\Gamma^{n+1} a_{0},\left[Q, a_{1}\right], \ldots\left[Q, a_{n}\right]\right) .
$$

For future reference, we also define the following sequence of multilinear functionals:

$$
\tilde{\tau}_{n}^{\beta}\left(a_{0}, a_{1}, \ldots, a_{n}\right):=F_{n}^{\beta}\left(\Gamma^{n} a_{0},\left[Q, a_{1}\right], \ldots\left[Q, a_{n}\right]\right) .
$$

II.B. Let $\mathbb{R}_{+}^{1 \mid 1}$ denote the (1|1)-dimensional superdomain whose base is the set of positive real numbers. The coproduct morphism $\Delta: C^{\infty}\left(\mathbb{R}_{+}^{1 \mid 1}\right) \rightarrow C^{\infty}\left(\mathbb{R}_{+}^{1 \mid 1}\right) \widehat{\otimes}_{\pi}$ $C^{\infty}\left(\mathbb{R}_{+}^{1 \mid 1}\right)$ given by

$$
(\Delta f)(s, \delta ; t, \varepsilon):=f(s+t-\delta \varepsilon, \delta+\varepsilon),
$$

and the counit morphism $\imath: \mathscr{C}^{\infty}\left(\mathbb{R}_{+}^{1 \mid 1}\right) \rightarrow \mathbb{R}$ given by

$$
\text { If }:=f(0,0)
$$

furnish $\mathbb{R}_{+}^{1 \mid 1}$ with the structure of supersemigroup. Informally, we will use the product notation, $(s, \delta) \cdot(t, \varepsilon)=(s+t-\delta \varepsilon, \delta+\varepsilon)$.

Proposition II.1 The morphism $\mathscr{C}^{\infty}\left(\mathbb{R}_{+}^{1 \mid 1}\right) \rightarrow \mathscr{C}^{\infty}\left(\mathbb{R}_{+}^{1 \mid 1}\right) \widehat{\otimes}_{\pi} \mathscr{L}(\mathscr{H})$ given by

$$
(t, \varepsilon) \rightarrow T(t, \varepsilon):=\exp \left(-t Q^{2}+\varepsilon Q\right)
$$

defines a representation of $\mathbb{R}_{+}^{1 \mid 1}$ on $\mathscr{H}$.

Proof. We note that

$$
\begin{aligned}
\exp \delta Q \exp \varepsilon Q & =(1+\delta Q)(1+\varepsilon Q) \\
& =1+(\delta+\varepsilon) Q+\delta \varepsilon Q^{2}=\exp \left(\delta \varepsilon Q^{2}+(\delta+\varepsilon) Q\right),
\end{aligned}
$$

and so

$$
\begin{aligned}
T(s, \delta) T(t, \varepsilon) & =\exp \left(-s Q^{2}+\delta Q\right) \exp \left(-t Q^{2}+\varepsilon Q\right) \\
& =\exp \left(-(t+s-\delta \varepsilon) Q^{2}+(\delta+\varepsilon) Q\right)=T((s, \delta) \cdot(t, \varepsilon)),
\end{aligned}
$$

and the claim follows.

II.C. For a positive integer $n$ and $\beta>0$, we define the $(n \mid n)$-supersimplex $\sigma_{n \mid n}^{\beta}$ to be an $(n \mid n)$-dimensional superdomain [1] whose base is the $n$-simplex $\sigma_{n}^{\beta}$. We let $t_{1}, \ldots, t_{n}$ and $\varepsilon_{1}, \ldots, \varepsilon_{n}$ denote the even and odd generators of the structure sheaf of $\sigma_{n \mid n}^{\beta}$, respectively. By $\int_{\sigma_{n \mid n}^{\beta}} \ldots d t d \varepsilon$ we denote the usual Berezin integral [1].

We set for $a \in \mathscr{A}$ and $(t, \varepsilon) \in \sigma_{1 \mid 1}^{\beta}$,

$$
a(t, \varepsilon):=\exp \left(-t Q^{2}+\varepsilon Q\right) a \exp \left(t Q^{2}-\varepsilon Q\right),
$$

We note that $a(t)=a(t, 0)$. 
Proposition II.2 We have the following identities:

(i)

$$
\int a(t, \varepsilon) d \varepsilon=[Q, a](t) ;
$$

(ii) As operators on $\operatorname{Ran}\left(\exp \left(-\beta Q^{2}\right)\right)$,

$$
\exp \left(-s Q^{2}+\delta Q\right) a(t, \varepsilon) \exp \left(s Q^{2}-\delta Q\right)=a((s, \delta) \cdot(t, \varepsilon)) .
$$

Proof. The first identity follows from the observation that

$$
a(t, \varepsilon)=a(t)+\varepsilon \exp \left(-t Q^{2}\right)[Q, a] \exp t Q^{2} .
$$

The proof of the second identity is similar to the proof of Proposition II.1.

II.D. An immediate consequence of (II.2) and Proposition II.2 (i) is the following representation of the Chern charater by means of a Berezin integeral over the supersimplex $\sigma_{n \mid n}^{\beta}$,

$$
\tau_{n}^{\beta}\left(a_{0}, a_{1}, \ldots, a_{n}\right)=\beta^{-n / 2} \int_{\substack{\sigma_{n \mid n}^{\beta} \\ \sigma_{n \mid n}}} \operatorname{Tr}\left(\Gamma^{n+1} a_{0} a_{1}\left(t_{1}, \varepsilon_{1}\right) \ldots a_{n}\left(t_{n}, \varepsilon_{n}\right) \exp \left(-\beta Q^{2}\right)\right) d t d \varepsilon
$$

A similar representation holds for $\tilde{\tau}_{n}^{\beta}$,

$$
\tilde{\tau}_{n}^{\beta}\left(a_{0}, a_{1}, \ldots, a_{n}\right)=\beta^{-n / 2} \int_{\sigma_{n \mid n}^{\beta}} \operatorname{Tr}\left(\Gamma^{n} a_{0} a_{1}\left(t_{1}, \varepsilon_{1}\right) \ldots a_{n}\left(t_{n}, \varepsilon_{n}\right) \exp \left(-\beta Q^{2}\right)\right) d t d \varepsilon .
$$

\section{Translation Invariance in Superspace and Connes' Coboundary}

An immediate consequence of the representation (II.11), and the cyclicity of the trace is the following translation invariance property.

Proposition III.1. The functional $\tilde{\tau}_{n}^{\beta}$ is invariant under the supersemigroup $\mathbb{R}_{+}^{1 / 1}$,

$$
\tilde{\tau}_{n}^{\beta}\left(a_{0}(s, \eta), a_{1}(s, \eta), \ldots, a_{n}(s, \eta)\right)=\tilde{\tau}_{n}^{\beta}\left(a_{0}, a_{1}, \ldots, a_{n}\right) .
$$

Let us now take the left derivative $\partial / \partial_{\eta}$ of (III.1) and evaluate it at $s=0, \eta=0$. Keeping in mind the sign count in the super Leibniz rule, we obtain the equation

$$
\sum_{0 \leqq j \leqq n}(-1)^{j} \tilde{\tau}_{n}^{\beta}\left(a_{0}, \ldots,\left[Q, a_{j}\right], \ldots, a_{n}\right)=0
$$

We claim that the above identity is just the cocycle condition $\partial \tau^{\beta}=0$, where $\partial=B+b$ is Connes' coboundary operator of entire cyclic cohomology (see [2] for the definitions of $b$ and $B$; below we use the normalization of [7]). This follows from the proposition formulated below. The proof of this proposition can be easily extracted from the computation on pages 12-13 of [7]. For completeness, we present a superspace version of these computations. 
Proposition III.2. We have the identities

$$
\left(B \tau^{\beta}\right)_{n}\left(a_{0}, a_{1}, \ldots, a_{n}\right)=\beta^{1 / 2} \tilde{\tau}_{n}^{\beta}\left(\left[Q, a_{0}\right], a_{1}, \ldots, a_{n}\right)
$$

and

$$
\left(b \tau^{\beta}\right)_{n}\left(a_{0}, a_{1}, \ldots, a_{n}\right)=\beta^{1 / 2} \sum_{1 \leqq j \leqq n}(-1)^{j} \tilde{\tau}_{n}^{\beta}\left(a_{0}, \ldots,\left[Q, a_{j}\right], \ldots, a_{n}\right)
$$

Proof. The proof of (III.3) is a straightforward calculation. Using cyclicity of the trace, we find

$$
\begin{aligned}
& \beta^{(n+1) / 2}\left(B \tau^{\beta}\right)_{n}\left(a_{0}, a_{1}, \ldots, a_{n}\right) \\
& =\beta^{(n+1) / 2} \sum_{j=0}^{n}(-1)^{n j} \tau_{n+1}^{\beta}\left(I, a_{n+1-j}, \ldots, a_{n}, a_{0}, \ldots, a_{n-j}\right) \\
& =\sum_{j=0}^{n}(-1)^{n j} \int_{\sigma_{n+1 \mid n+1}^{\beta}} \operatorname{Tr}\left(\Gamma^{n} a_{n+1-j}\left(t_{1}, \varepsilon_{1}\right) \ldots\right. \\
& \left.\quad a_{n}\left(t_{j-1}, \varepsilon_{j-1}\right) a_{0}\left(t_{j}, \varepsilon_{j}\right) \ldots a_{n-j}\left(t_{n-j}, \varepsilon_{n-j}\right) e^{-\beta Q^{2}}\right) d t d \varepsilon \\
& =\sum_{j=0}^{n} \int_{\sigma_{n+1 \mid n+1}^{\beta}} \operatorname{Tr}\left(\Gamma^{n} a_{0}\left(0, \varepsilon_{0}\right) \ldots a_{j}\left(t_{j}, \varepsilon_{j}\right) a_{j+1}\left(t_{j+2}, \varepsilon_{j+1}\right) \ldots\right. \\
& \left.a_{n}\left(t_{n+1}, \varepsilon_{n}\right) e^{-\beta Q^{2}}\right) d t d \varepsilon,
\end{aligned}
$$

using translation invariance in $t$. Integrating out the redundant variables, we rewrite the above expression as

$$
\begin{aligned}
& \sum_{j=0}^{n} \int_{\sigma_{n \mid n}^{\beta}}\left(t_{j+1}-t_{\jmath}\right) \operatorname{Tr}\left(\Gamma^{n}\left[Q, a_{0}\right] a_{1}\left(t_{1}, \varepsilon_{1}\right) \ldots a_{n}\left(t_{n}, \varepsilon_{n}\right) \exp \left(-\beta Q^{2}\right)\right) d t d \varepsilon \\
& \quad=\beta \int_{\substack{\sigma_{n \mid n}^{\beta}\\
}} \operatorname{Tr}\left(\Gamma^{n}\left[Q, a_{0}\right] a_{1}\left(t_{1}, \varepsilon_{1}\right) \ldots a_{n}\left(t_{n}, \varepsilon_{n}\right) \exp \left(-\beta Q^{2}\right)\right) d t d \varepsilon \\
& =\beta^{(n+2) / 2} \tilde{\tau}_{n}^{\beta}\left(\left[Q, a_{0}\right], a_{1}, \ldots, a_{n}\right),
\end{aligned}
$$

where $t_{0}:=0, t_{n+1}:=\beta$, and our assertion is proven.

We now prove (III.4). Using Proposition II.2 (ii), we obtain the representation

$$
\begin{aligned}
& \beta^{n / 2} \tilde{\tau}_{n}^{\beta}\left(a_{0}, a_{1}, \ldots,\left[Q, a_{j}\right], \ldots, a_{n}\right) \\
& \quad=\iint_{\substack{\sigma_{n \mid n}^{\beta} \\
n}} \operatorname{Tr}\left(\Gamma^{n} a_{0} a_{1}\left(t_{1}, \varepsilon_{1}\right) \ldots a_{j}\left(t_{j}-\delta \varepsilon_{j}, \varepsilon_{j}+\delta\right) \ldots a_{n}\left(t_{n}, \varepsilon_{n}\right) \exp \left(-\beta Q^{2}\right)\right) d t d \varepsilon d \delta .
\end{aligned}
$$

But $a_{j}\left(t_{j}-\delta \varepsilon_{j}, \varepsilon_{J}+\delta\right)=a_{j}\left(t_{j}-\delta \varepsilon_{j}, 0\right)+\left(\varepsilon_{j}+\delta\right)\left[a_{j}, Q\right]\left(t_{j}\right)=a_{j}\left(t_{j}-\delta \varepsilon_{j}\right)+\left(\varepsilon_{j}+\right.$ $\delta)\left[a_{j}, Q\right]\left(t_{j}\right)$, because of $\varepsilon_{j}^{2}=\delta^{2}=0$. The term proportional to $\varepsilon_{j}+\delta$ gives a zero contribution to (III.5), and so we are left with $a_{j}\left(t_{j}-\delta \varepsilon_{j}\right)$ only. At this point one is tempted to make a change of variables $t_{j}-\delta \varepsilon_{j} \rightarrow t_{j}$ to conclude that the integral 
is zero. This, however, is incorrect, as the integrand in (III.5) is not compactly supported [1]. Instead, we have for $j=1,2, \ldots, n$,

$$
\int a_{J}\left(t_{j}-\delta \varepsilon_{j}\right) d t_{j} d \varepsilon_{j} d \delta=-\int_{t_{j-1}}^{t_{j+1}} \frac{d}{d t_{j}} a_{j}\left(t_{j}\right) d t_{j}=a_{j}\left(t_{j-1}\right)-a_{j}\left(t_{j+1}\right)
$$

(we set here $t_{0}:=0, t_{n+1}:=\beta$, and $\varepsilon_{0}=\varepsilon_{n+1}:=0$ ) and consequnetly

$$
\begin{aligned}
& \beta^{n / 2} \tilde{\tau}_{n}^{\beta}\left(a_{0}, a_{1}, \ldots,\left[Q, a_{j}\right], \ldots, a_{n}\right) \\
& =-\int_{\sigma_{n-1 \mid n-1}^{\beta}} \operatorname{Tr}\left(\Gamma^{n} a_{0} a_{1}\left(t_{1}, \varepsilon_{1}\right) \ldots a_{j}\left(t_{j}\right) a_{j+1}\left(t_{j}, \varepsilon_{j}\right) \ldots a_{n}\left(t_{n}, \varepsilon_{n}\right) e^{-\beta Q^{2}}\right) d t d \varepsilon \\
& +\int_{\sigma_{n-1 \mid n-1}^{\beta}} \operatorname{Tr}\left(\Gamma^{n} a_{0} a_{1}\left(t_{1}, \varepsilon_{1}\right) \ldots a_{j-1}\left(t_{j-1}, \varepsilon_{j-1}\right) a_{j}\left(t_{j-1}\right) \ldots\right. \\
& \left.\quad a_{n}\left(t_{n-1}, \varepsilon_{n-1}\right) e^{-\beta Q^{2}}\right) d t d \varepsilon .
\end{aligned}
$$

Using the fact that

$$
\left(a_{1} a_{2}\right)(t, \varepsilon)=a_{1}(t, \varepsilon) a_{2}(t)+a_{1}(t) a_{2}(t, \varepsilon),
$$

we thus obtain

$$
\begin{aligned}
& \beta^{n / 2} \sum_{1 \leqq j \leqq n}(-1)^{j} \tilde{\tau}_{n}^{\beta}\left(a_{0}, a_{1}, \ldots,\left[Q, a_{j}\right], \ldots, a_{n}\right) \\
& =\sum_{j=0}^{n-1}(-1)^{j} \int_{\sigma_{n-1 \mid n-1}^{\beta}} \operatorname{Tr}\left(\Gamma^{n} a_{0} a_{1}\left(t_{1}, \varepsilon_{1}\right) \ldots\left(a_{j} a_{j+1}\right)\left(t_{j}, \varepsilon_{j}\right) \ldots a_{n}\left(t_{n}, \varepsilon_{n}\right) e^{-\beta Q^{2}}\right) d t d \varepsilon \\
& =\beta^{(n-1) / 2}\left(b \tau^{\beta}\right)_{n}\left(a_{0}, a_{1}, \ldots, a_{n}\right)
\end{aligned}
$$

and the claim follows.

\section{Path Integral Representation of the Index}

$I V . A$. We now assume that the $\Theta$-summable module $(\mathscr{H}, Q, \mathscr{A})$ is associated with a supersymmetric quantum theory. This theory may involve finitely many degrees of freedom (quantum mechanics) or an infinite number of degrees of freedom (quantum field theory). As a rule, a quantum theory involving finitely many degrees of freedom leads to a p-summable Fredholm module with the associated dimension equal to the number of degrees of freedom. Quantum field theories lead to infinite dimensional $\Theta$-summable Fredholm modules. Aside from some simple examples, the infinite dimensional constructions described below have not been yet carried through in a rigorous manner. Therefore, in mathematical terms, the results formulated below have a partially conjectural character as they rely on the existence and properties of certain measures on infinite dimensional Grassmann algebras.

More specifically, we require the existence of a Euclidean supersymmetric quantum field theory in the following strong sense. 
- The algebra $\mathscr{A}$ consists of suitable functions of "time zero bosonic and fermionic field operators" $\varphi_{j}(x)$ and $\psi_{j}(x)$, where $j=1, \ldots, n$. Here $x \in \Sigma$, where $\Sigma$ is a compact Riemannian manifold. In the case of quantum mechanics, $\Sigma$ consists of a single point. We will assume that $\varphi_{j}(x)$ is a real scalar field, while $\psi_{j}(x)$ is a Majorana Fermi field. In fact, these are operator valued distributions, and so only smoothed out objects $\varphi_{j}(f)$ and $\psi_{j}(f)$ (with $f$ a test function) are well defined operators. The fact that the number of bosonic operators is set to be equal to the number of fermionic operators is not accidental: it reflects the underlying symmetry (supersymmetry) of the theory. A natural way to describe a supersymmetric theory is the language of superfields.

- There exists an underlying space of Euclidean superfields. Euclidean superfields are integration variables in an infinite dimensional Berezin integral, very much like Brownian paths are integration variables in a Wiener integral. For our needs, a scalar superfield $\Phi_{j}(x, t, \varepsilon)((x, t) \in \Sigma \times \mathbb{R}), j=1, \ldots, n$, has the form $\Phi_{j}(x, t, \varepsilon)=\phi_{j}(x, t)+\varepsilon \Psi_{j}(x, t)$, where $\phi_{j}(x, t)$ is a Euclidean bosonic field, and where $\Phi_{j}(x, t)$ is a Euclidean Majorana field. Strictly speaking, Euclidean superfields are Grassmann algebra valued distributions, and so need to be regularized to produce non-singular objects. Observe that the algebra generated by the regularized superfields is commutative; this is a consequence of the fact that Euclidean Bose fields commute and Euclidean Fermi fields anticommute.

- The key elements of quantum theory are various Feynman-Kac formulas which provide a bridge between the Hilbert space and Euclidean formulations of the theory. A Feynman-Kac formula relates a functional on the algebra $\mathscr{A}$ (like the vacuum value expectation, trace or supertrace) to an integral over $\mathscr{M}$ with respect to a suitable measure $d \mu(\Phi)$. For our purposes we require the following FeynmanKac formula. For $a_{0}, \ldots, a_{n} \in \mathscr{A}$ and $(t, \varepsilon) \in \sigma_{n \mid n}^{\beta}$, there exist functions $A_{0}, \ldots, A_{n}$ of $\Phi(\cdot, t, \varepsilon)$ such that

$$
\operatorname{Tr}\left(\Gamma a_{0}\left(t_{0}, \varepsilon_{0}\right) a_{1}\left(t_{1}, \varepsilon_{1}\right) \ldots a_{n}\left(t_{n}, \varepsilon_{n}\right) \exp \left(-\beta Q^{2}\right)\right)=\int \prod_{j=0}^{n} A_{j}\left(\Phi\left(\cdot, t_{j}, \varepsilon_{j}\right)\right) d \mu^{\beta}(\Phi)
$$

where $d \mu^{\beta}(\Phi)$ is a measure depending on $\beta$. In concrete physical models, $\beta$ dependence means that the measure $d \mu^{\beta}(\Phi)$ is concentrated on $\Phi$ 's which are periodic in $t$ with period $\beta$.

$I V . B$. Assuming the existence of the structure described in the previous subsection we can prove the following theorem. It expresses the index ind $\left(Q_{p+}\right)$ of the operator $Q_{p}:=p Q p$, where $p \in \mathscr{A}$ is a projection, as a supersymmetric path integral. This identity generalizes the local version of the Atiyah-Singer index theorem; for more background and motivation see [2] and [3].

Theorem IV.1. Let $P$ be a function of $\Phi$ corresponding to $p$. then,

$$
\begin{aligned}
\operatorname{ind}\left(Q_{p+}\right)= & \int P(\Phi(\cdot, 0,0)) \exp \left[-\beta^{-1}\left(\int P(\Phi(\cdot, t, \varepsilon)) d t d \varepsilon\right)^{2}\right] d \mu^{\beta}(\Phi) \\
& +\frac{1}{2} \int\left\{1-\exp \left[-\beta^{-1}\left(\int P(\Phi(\cdot, t, \varepsilon)) d t d \varepsilon\right)^{2}\right]\right\} d \mu^{\beta}(\Phi)
\end{aligned}
$$


Proof. According to the results of [3] and [6], the index ind $\left(Q_{p+}\right)$ can be computed from the even part of the Chern character according to the formula

$$
\operatorname{ind}\left(Q_{p+}=\tau_{0}^{\beta}(p)+\sum_{k=1}^{\infty}(-1)^{k} \frac{(2 k) !}{k !} \tau_{2 k}^{\beta}\left(p-\frac{1}{2}, p, \ldots, p\right)\right. \text {. }
$$

From (IV.1) and (II.10),

$$
\begin{aligned}
& \tau_{2 k}^{\beta}\left(a_{0}, a_{1}, \ldots, a_{2 k}\right) \\
& =\beta^{-k} \int_{\sigma_{2 k \mid 2 k}^{\beta}} A_{0}(\Phi(\cdot, 0,0)) A_{1}\left(\Phi\left(\cdot, t_{1}, \varepsilon_{1}\right)\right) \ldots A_{2 k}\left(\Phi\left(\cdot, t_{2 k}, \varepsilon_{2 k}\right)\right) d \mu^{\beta}(\Phi) d t d \varepsilon \\
& =(2 k) !^{-1} \beta^{-1} \int_{I_{2 k \mid 2 k}^{\beta}}^{\beta} \int A_{0}(\Phi(\cdot, 0,0)) \prod_{j=1}^{2 k} A_{j}\left(\Phi\left(\cdot, t_{j}, \varepsilon_{j}\right)\right) d \mu^{\beta}(\Phi) d t d \varepsilon,
\end{aligned}
$$

where we have used the fact that the Euclidean scalar superfields commute with each other, and where $I_{2 k \mid 2 k}^{\beta}$ denotes the $(2 k \mid 2 k)$-dimensional supercube of side $\beta$. Substituting this into (IV.3) we obtain

$$
\begin{aligned}
\operatorname{ind}\left(Q_{p+}\right) & =\int P(\Phi(\cdot, 0,0)) d \mu^{\beta}(\Phi) \\
& +\sum_{k=1}^{\infty} \frac{(-\beta)^{-k}}{k !} \int\left(P(\Phi(\cdot, 0,0))-\frac{1}{2}\right)\left(\int P(\Phi(\cdot, t, \varepsilon)) d t d \varepsilon\right)^{2 k} d \mu^{\beta}(\Phi),
\end{aligned}
$$

and the claim follows.

Acknowledgement. The work presented in this paper was done in January'93 while the first named author was visiting the Forschungainstitut für Mathematik at ETH Zürich, whose hospitality he would like to gratefully acknowledge.

\section{References}

1. Berezin, F.A.: Introduction to Superanalysis. Dordrecht, D. Reidel Publ. Co., (1987)

2. Connes, A.: Non-commutative differential geometry. Publ. Math. IHES 62, 94-144 (1986)

3. Connes, A.: Entire cyclic cohomology of Banach algebras and characters of $\theta$-summable Fredholm modules. K-Theory, 1, 519-548 (1988)

4. Connes, A., Moscovici, H.: Transgression and the Chern character in non-communitative Khomology. Commun. Math. Phys.155, 103-122 (1993)

5. Ernst, K., Feng, P., Jaffe, A., Lesniewski, A.: Quantum K-theory, II. Homotopy invariance of the Chern character. J. Funct. Anal. 90, 355-368 (1990)

6. Getzler, E., Szenes, A.: On the Chern character of a theta-summable Fredholm module. J. Funct. Anal. 84, 343-357 (1989)

7. Jaffe, A., Lesniewski, A., Osterwalder, K.: Quantum K-theory, I. The Chern character. Commun. Math. Phys. 118, 1-14 (1988)

9. Osterwalder, K., Schrader, R.: Euclidean Fermi fields and a Feynman-Kac formula for bosonfermion models. Helv. Phys, Acta 46, 277-302 (1973) 